\title{
Travailler Moins pour Travailler Plus Longtemps
}

\author{
Helmuth Cremer, ${ }^{1}$ Philippe De Donder, ${ }^{2}$ \\ Darío Maldonado ${ }^{3}$ et Pierre Pestieau ${ }^{4}$
}

Première version: Octobre 2008

Version révisée: Septembre 2010

1. Toulouse School of Economics (GREMAQ, IDEI et Institut universitaire de France)

2. Toulouse School of Economics (GREMAQ-CNRS et IDEI)

3. Department of Economics and CeiBA-Complejidad, Universidad del Rosario, Bogota

4. CREPP, HEC-Management School University of Liège; CORE, Université Catholique de Louvain; PSE et CEPR. 


\section{Résumé}

Nous développons un modèle dans lequel les travailleurs peuvent avoir tendance à trop travailler quand ils sont jeunes, parce qu'ils ne peuvent ou ne veulent pas voir les conséquences que cela peut avoir sur leur santé et sur leur capacité à travailler longtemps. Pour les obliger à mieux répartir leurs efforts sur leur cycle de vie, une taxe sur les revenus du travail en début de vie et une subvention de l'épargne sont souhaitables. Si la subvention de l'épargne n'est pas disponible, la taxe optimale arbitre entre quatre considérations différentes. Nous étudions également la taxe optimale quand les individus diffèrent en productivité. ${ }^{*}$

* Mots clés: Myopie, Retraite, Etat paternaliste, Biens peccamineux Codes JEL: D91, H21, H55 


\section{Introduction}

Depuis plusieurs décennies, on assiste à une baisse du taux d'activité des travailleurs âgés. Bien que depuis quelques années la tendance se soit inversée, nous avons aujourd'hui une durée moyenne de retraite inégalée et financièrement insoutenable et ce, d'autant que l'espérance de vie ne cesse de croître. Quelle est la cause de cette situation? Du côté offre, on incrimine en général deux facteurs: les effets désincitatifs des régimes de protection sociale et l'état de santé des travailleurs. Du côté de la demande, on invoque l'intérêt que les entreprises peuvent avoir à se débarrasser d'une main d'œuvre trop coûteuse pour ce qu'elle rapporte. On invoque aussi l'illusoire croyance en une relation comptable entre l'emploi des jeunes et celui des vieux: un travailleur âgé conduit à la retraite offrirait son emploi à un jeune chômeur.

Les travaux théoriques portant sur le départ à la retraite ont surtout mis l'accent sur les sanctions implicites qu'impliquaient les systèmes de retraite pour qui veut prolonger sa vie active. ${ }^{1}$ Ils ont négligé la question de la santé, sans doute parce que celleci était traitée comme exogène et non pas résultant de décisions individuelles prises antérieurement.

L'objet de cet article est précisément de mettre la santé au centre de la décision de cessation d'activité et de la traiter comme endogène. De nombreux travaux empiriques montrent que l'état de santé est une des variables déterminantes de la cessation d'activité des travailleurs âgés (Bound et al. [1999], Currie et Madrian [1999], Dwyer et Mitchell [1999], Kerkhofs et al. [1999][kerkhofs], Kreider [1999], Campolieti [2002]). Selon l'enquête SHARE menée en 2004 dans dix pays Européens, une proportion importante de personnes de 50 à 64 ans ayant un emploi souhaitent prendre leur retraite le plus rapidement possible: cette proportion varie de $30 \%$ aux Pays Bas à $67 \%$ en Espagne, en passant par 57\% en France. Dans le même temps, la proportion de seniors ayant encore un emploi qui déclarent leur travail physiquement pénible est également élevée: 39,8 \% en France contre 47,1 \% en moyenne pour les 10 pays étudiés. BLANChet et Debrand [2007] exploitent ces données et obtiennent que "les variables les

1. Voir Cremer et al. [2008] et aussi Fenge et Pestieau [2005] 
plus discriminantes vis-à-vis du souhait d'un départ rapide sont la satisfaction globale au travail et la crainte que des problèmes de santé ne limitent la capacité de travail avant l'âge normal de la retraite: [...] craindre d'être limité par un problème de santé accroît cette même probabilité de 14,3 points. [...] L'état de santé des individus est lui aussi corrélé avec ce souhait. Être en mauvais ou très mauvais état de santé est lié à une augmentation de ce dernier de plus de 11 points."

Debrand et Lengagne [2007] on également étudié la relation entre retraite anticipée et santé. Ils montrent que "la pénibilité et les problèmes de santé d'origine professionnelle se seraient accrus dans tous les secteurs d'activité. La pénibilité physique présente une tendance croissante, bien que les emplois du secteur primaire - souvent assimilés à de fortes contraintes de travail - se soient raréfiés. Les pénibilités psychologiques se sont également développées. Le stress au travail serait ainsi responsable d'une part croissante des problèmes de santé d'origine professionnelle." La pénibilité du travail semble particulièrement importante chez les travailleurs non qualifiés (CAROLI et Gautie, 2009).

La littérature (en ce compris Debrand et Lengagne [2007]) a étudié les liens existant entre l'organisation du travail et la santé des seniors en se fondant sur deux modèles, celui de Karasek et Theorell [1991] et celui de Siegrist [1996] qui mettent en avant trois dimensions: le niveau de pression ressenti, la latitude décisionnelle laissée au travailleur, et le sentiment d'être récompensé pour son travail. En revanche, la relation entre durée de travail excessive et santé a été beaucoup moins étudiée, sauf aux Etats-Unis qui disposent d'excellentes données longitudinales. VAN DER HULST [2003] et DAhlgren [2006] montrent l'effet désastreux que l'excès de travail peut avoir sur la santé, via une augmentation de la vulnérabilité aux maladies liées au stress, comme les maladies cardiaques, le diabète ou l'obésité. Plus spécifiquement, Dembe et al. [2005] montrent que la fréquence d'accidents de travail est $61 \%$ plus élevée chez ceux qui font des heures supplémentaires. L'OCDE commence également à s'intéresser à ces questions: un rapport récent (voir OECD [2008]) indique ainsi que "mental health appears to have worsened in certain countries and for certain workforce groups, such as low-skilled workers. The reported incidence of certain potentially stressful working conditions has also 
increased (e.g. high-intensity work and long working hours)".

L'idée selon laquelle certains individus "se tuent à la tâche" peut faire sourire dans un pays connu pour ses RTT et ses 35 heures. Dans l'OCDE, la France est un des pays où la durée du temps de travail est la plus basse. Le nombre d'heures de travail annuelles par habitant varie de 611 pour la France à 1120 pour la Corée. Si l'on prend le nombre d'heures par travailleur, cela va de 1340 pour les Pays Bas à 2410 pour la Corée (1420 pour la France). A cette objection facile, on répondra en deux points. D'abord, la portée de notre article dépasse la France. Ensuite, ces chiffres sont des moyennes. En France comme ailleurs, une fraction des travailleurs fait des heures supplémentaires, travaille le week-end et pendant les vacances. C'est cette fraction qui nous intéresse. Selon l'OCDE [2004], la distribution de la durée de travail hebdomadaire en France connaît deux pointes: la plus importante à $35 \mathrm{~h}$ et l'autre à $45 \mathrm{~h}$. Selon le troisième survey européen sur les conditions de travail (BOISARD et al. [2002]), 17\% des employés à temps complet travaillent plus de 45 heures par semaines. Cette proportion est de $30 \%$ en Suède (DAhlgren [2006]). C'est à cette fraction de la population qui s'expose à des problèmes de santé, lesquels conduisent à une cessation d'activité précoce, que nous nous intéressons.

On peut se demander pourquoi choisir un rythme de travail qui conduit à raccourcir la vie active. Il y a sans doute des contraintes de liquidité: les jeunes actifs peuvent difficilement emprunter sur l'avenir pour financer des besoins immédiats. Il peut aussi y avoir un mélange d'ignorance et de myopie. C'est de cette question que traite cet article. Il s'inscrit dans la ligne des travaux sur les biens peccamineux ("sin goods"), biens dont la consommation a un effet néfaste sur la santé, effet souvent décalé dans le temps et ignoré par les individus (voir O’Donoghue et RABin [2003, 2006] et Cremer et al. [2010]). L'introduction de considérations d'inconsistance temporelle des préférences, de myopie et de procrastination est récente dans la littérature économique sur les retraites, dans laquelle les considérations de redistribution et d'efficacité dynamique ont longtemps prévalu (voir De Donder [2007] pour un rapide tour d'horizon). Ce type de phénomènes est pourtant bien connu, ne serait-ce qu'au niveau de l'épargne. ${ }^{2}$ Notre objectif ici est

2. Cette idée remonte à Strotz [1956]. 
d'étudier les conséquences pour un individu de la myopie dont il peut faire preuve au moment de choisir l'ampleur de son offre de travail, et d'analyser comment la politique fiscale peut aider à corriger ces erreurs.

Nous développons un modèle dans lequel les travailleurs vivent deux périodes. Dans la première période, ils peuvent choisir un volume de travail élevé qui leur apporte des revenus immédiats, mais qui a des conséquences néfastes sur leur santé au bout de quelque temps. Les travailleurs ne voient pas ces conséquences négatives, par ignorance ou par myopie. Outre la décision du volume de travail, les agents décident également du montant de leur épargne. Dans la seconde partie de leur vie active, les individus découvrent les conséquences sur leur santé de leur choix de travail de première période, et réagissent en optimisant à la fois leur volume de travail (l'âge de départ à la retraite, par exemple) et le montant des soins de santé dans lesquels ils souhaitent investir.

Il y a plusieurs manières de travailler "trop": au travers des heures supplémentaires, ou aussi d'un second boulot ou du travail au noir en fin de journée ou de semaine, ou encore par un travail physiquement pénible ou psychologiquement stressant. Dans cet article, nous ne traitons que de la durée du travail marchand qui peut être régulée par une taxe linéaire sur les salaires. Nous ne nous intéressons pas au travail au noir ni au travail domestique et encore moins à la pénibilité dont la régulation est beaucoup plus difficile, si ce n'est impossible. Le travail au noir demande un contrôle accru; les conditions de travail par trop pénibles appellent une réglementation légale et réguler le travail domestique est quasiment impossible. ${ }^{3}$

Dans cet article, nous supposons qu'un travail long et pénible affecte la capacité à travailler plus ou moins longtemps en seconde période mais pas la durée (espérée) de cette seconde période. Endogénéiser la durée de cette seconde période (ou la probabilité d'être en vie en seconde période) constituerait une extension pertinente de notre modèle. Il est connu qu'un excès de travail dans les jeunes années peut avoir un effet négatif sur la longévité. Si cet effet n'est pas pris en compte en première période, les individus

3. Remarquons que les erreurs commises en première période peuvent être dues à une myopie tout comme à un manque d'information. Dans les deux cas, l'individu observe la réalité de son état de santé en seconde période et regrette ses choix de première période. Dans la seconde interprétation, une action bénéfique de l'autorité publique consisterait à disséminer l'information concernant le lien entre travail excessif et santé à long terme. 
peuvent se retrouver avec une seconde période plus courte qu'ils ne l'avaient anticipée. Dans cette hypothèse, ils seraient sans doute amenés à prendre leur retraite plus tôt que prévu et à consommer plus en seconde période qu'ils ne l'avaient anticipé. Nous sommes conscients que l'hypothèse d'exogénéité de l'espérance de vie que nous faisons ici est forte surtout lorsque l'on parle de retraite. Il existe une très vaste littérature ${ }^{4}$ sur les anticipations d'espérance de vie et la retraite. Mais cette dimension du problème aurait considérablement compliqué et allongé cet article.

Anticipant sur la suite de cet article, nous en donnons dès à présent les principaux résultats en commençant par un monde de laissez-faire dans lequel les individus sont tous identiques. Nous montrons que les agents travaillent trop en première période, car ils n'anticipent pas les conséquences de cet excès de travail sur leur état de santé futur. En revanche, leur myopie engendre deux effets contraires sur le montant de leur épargne. D'une part, comme ils travaillent trop, ils épargnent également plus que ce qui serait optimal. D'un autre côté, ils sous-estiment la pénibilité du travail de seconde période (car ils surestiment leur état de santé) et leurs besoins médicaux, ce qui les pousse à épargner trop peu. Dans une situation de premier rang, un planificateur utilitariste a donc besoin de deux instruments pour décentraliser l'allocation optimale: une taxe sur le travail de première période assortie d'une subvention à l'épargne.

Nous étudions ensuite la situation de second rang dans laquelle le planificateur ne dispose que de la taxe sur le travail (par exemple, parce que l'épargne n'est pas observable), dont le produit est redistribué sous forme de retraite. Dans ce cas, la taxe qui décentralise l'offre optimale de travail en première période pousse les individus à épargner trop peu (car seul le second des deux effets mentionnés ci-dessus est alors présent). Nous montrons que la taxe optimale de second rang arbitre entre quatre considérations. Deux d'entre elles poussent à une taxe élevée. D'une part, la taxe transfère du pouvoir d'achat vers la seconde période, avec une utilité marginale plus élevée qu'en première période. D'autre part, la taxe décourage le travail de première période et diminue donc la désutilité du travail de seconde période. Une troisième considération pousse toujours à diminuer la taxe: il s'agit de la distorsion classique sur l'offre de travail. Le dernier effet a trait à

4. Voir à ce propos Hurd et MCGARRY [1995], HuRd et al [2004] et O'Donnell et al. [2008]. 
l'impact de la taxe sur le niveau d'épargne. Nous montrons que, si ce dernier effet est positif, alors la taxe optimale de second rang est toujours positive.

Nous analysons également le cas où les individus diffèrent en productivité. Le planificateur utilitariste a donc une raison supplémentaire de taxer les travailleurs, à savoir la redistribution. Cette raison supplémentaire tend à accroître la taxe optimale par rapport à la situation dans laquelle les individus ont une productivité identique. Si on compare notre modèle à un modèle classique de redistribution (sans myopie), la formule de taxation optimale comporte deux termes supplémentaires: un terme "pigouvien" de correction des "effets internes" qu'un travailleur s'inflige à lui-même, et un terme qui reflète le besoin d'égaliser par l'épargne l'utilité marginale des deux périodes.

\section{Le Modèle de Base: Un Seul Individu}

\section{II-1 Optimum de Premier Rang}

Nous étudions un individu de productivité $w$ qui travaille $\ell$ en première période et $z$ en seconde période: $\ell$ représente le nombre d'heures par semaine alors que $z$ peut inclure la partie de la seconde période qui est consacrée à l'activité professionnelle. ${ }^{5}$ L'individu épargne $s$ pour sa consommation de seconde période et son choix de $\ell$ et $z$ dépend de sa capacité à l'effort exprimée en termes quadratiques. En première période, il est en bonne santé alors qu'en seconde période son état de santé est plus ou moins bon et affecte sa décision de travailler. Cet état de "mauvaise santé", $h(\ell, e)$, dépend de deux variables: l'intensité de l'effort en première période, $\ell$, et un investissement en soins de santé effectué en seconde période, $e$. Une taxe linéaire, $\tau$, est imposée. Pour des raisons de simplicité, la taxe ne s'applique qu'au revenu de première période. Cette taxe permet de financer une prestation de retraite uniforme, $b,{ }^{6}$ mais aussi d'influencer le choix de $\ell$. En effet, nous faisons l'hypothèse que l'individu ignore ou ne veut pas voir l'effet que son choix de $\ell$ peut avoir sur son état de santé en seconde période et partant sur son choix de $z$.

Nous faisons l'hypothèse que l'utilité est additive entre périodes, que l'individu n'es-

5 . Si $\bar{\ell}$ dénote la durée de la semaine de travail et $k$ la fraction de la seconde période pendant laquelle l'individu est actif, on écrira: $z=\bar{\ell} k$. 
compte pas son utilité future et que le taux d'intérêt est nul, de sorte que son utilité totale s'écrit

$$
u\left(w \ell(1-\tau)-s-\frac{\ell^{2}}{2}\right)+u\left(w z-\frac{h(\alpha \ell, e) z^{2}}{2}-e+s+b\right),
$$

où $h_{2}(\alpha \ell, e)<0$ et $h_{1}(\alpha \ell, e)>0 ;^{7} \alpha=0$ en cas de myopie et $\alpha=1$ pour l'individu rationnel.

L'individu fait preuve de myopie en première période, au moment de choisir $\ell$. Cependant, en seconde période, il se rend compte de son erreur et adopte une valeur de $\alpha$ unitaire dans son choix de soins de santé. Notre Etat paternaliste essaie d'inciter l'individu à faire un choix rationnel dès la première période.

En première période, l'individu choisit $\ell$ et $s$, en se basant sur ce qu'il pense être son comportement futur en termes de choix de $z$ et de $e$. Les conditions du premier ordre (CPO) s'écrivent:

$$
\begin{aligned}
& u^{\prime}(\bar{c})=u^{\prime}\left(\bar{d}^{p}\right), \\
& \ell=w(1-\tau)-\frac{\alpha h_{1}\left(\alpha \ell, e^{p}\right)\left(z^{p}\right)^{2}}{2},
\end{aligned}
$$

où $\bar{c}$ et $\bar{d}$ dénotent les consommations $c$ et $d$ nettes de la désutilité du travail, et où l'exposant $p$ indique qu'il s'agit de la valeur prévue, du point de vue de la première période, d'une décision à prendre en seconde période. L'équation (1) repose sur l'hypothèse qu'il n'y a pas de contrainte de liquidité. Comme l'individu est myope en première période, l'équation (2) se réduit à $\ell=w(1-\tau)$ avec $\alpha=0$. Les valeurs prévues de $z$ et de $e$ sont données par les CPO

$$
\begin{aligned}
& z^{p}=\frac{w}{h\left(0, e^{p}\right)}, \\
& -\frac{h_{2}\left(0, e^{p}\right)\left(z^{p}\right)^{2}}{2}=1
\end{aligned}
$$

avec $\alpha=0$.

En seconde période, l'individu réalise l'impact de son choix de $\ell$ sur son état de santé

7. Nous dénotons la dérivée partielle de la fonction $h($.$) par rapport à son ième argument par h_{i}($.$) .$ 
et réoptimise en conséquence par rapport à $e$ et à $z$. Les CPO s'écrivent alors

$$
\begin{aligned}
& z=\frac{w}{h(\ell, e)} \\
& -\frac{h_{2}(\ell, e) z^{2}}{2}=1 .
\end{aligned}
$$

On peut comparer ces choix individuels avec les solutions optimales. Celles-ci viennent de la maximisation de l'expression suivante:

$$
\mathcal{L}=u\left(c-\frac{\ell^{2}}{2}\right)+u\left(d-\frac{h(\ell, e) z^{2}}{2}\right)-\mu[c+d+e-w(\ell+z)] .
$$

On obtient alors:

$$
\begin{aligned}
& u^{\prime}(\bar{c})=u^{\prime}(\bar{d})=-\frac{u^{\prime}(\bar{d}) h_{2}(\ell, e) z^{2}}{2}=\mu \\
& w=z h(\ell, e)=\ell+\frac{h_{1}(\ell, e) z^{2}}{2} .
\end{aligned}
$$

Comparons le laissez-faire (dénoté $L F$, obtenu en posant $\tau=b=0$ dans les équations (1)-(6)) et l'optimum social (dénoté par * et obtenu à partir des équations (7)-(8)). La comparaison de (2) et de (8) nous montre de suite que l'individu travaille trop en première période $\left(\ell^{L F}>\ell^{*}\right)$ car il sous-estime l'impact de ce travail sur sa santé future. En revanche, l'impact de sa myopie sur son niveau d'épargne est ambigu: d'un côté, son excès de travail de première période et les revenus qu'il en tire le poussent à épargner plus qu'il n'est optimal. D'un autre côté, la sous-estimation de ses besoins de santé de seconde période (et donc de son utilité marginale à ce moment) le conduit à épargner trop peu. On ne peut donc pas classer de façon non ambiguë sa consommation nette de première période dans les deux solutions:

$$
\bar{c}^{L F} \gtrless \bar{c}^{*}
$$

De ce fait, il n'est pas possible de décentraliser l'optimum à l'aide de nos deux instruments. En effet, nous ne disposons que d'un seul degré de liberté (le montant de la taxe $\tau$ détermine mécaniquement le montant de la retraite $b$ versée) alors qu'il nous en faudrait deux, l'un agissant sur l'offre de travail et l'autre sur le choix d'épargne. Certes, 
la taxe $\tau$ peut nous donner l'offre de travail optimale en première période. Pour $\alpha=0$, cette taxe est la solution de

$$
\ell=w(1-\tau)=\ell^{*}=w-h_{1}\left(\ell^{*}, e^{*}\right) \frac{\left(z^{*}\right)^{2}}{2} .
$$

Mais alors, comme l'individu ne perçoit pas l'effet négatif de son offre de travail en première période sur son offre de travail en seconde période, il aura tendance à ne pas épargner suffisamment. Une subvention à l'épargne $\theta$ s'impose afin d'obtenir

$$
\begin{aligned}
u^{\prime}\left(\frac{w^{2}(1-\tau)^{2}}{2}-s(1-\theta)\right)(1-\theta) & =u^{\prime}\left(\bar{d}^{*}\right) \\
& =u^{\prime}\left(w z^{*}+s+b-e^{*}-h\left(\ell^{*}, e^{*}\right) \frac{\left(z^{*}\right)^{2}}{2}\right),
\end{aligned}
$$

c'est-à-dire de sorte à égaliser la désutilité marginale de l'épargne perçue par l'individu en première période (le membre de gauche de (9)) à son niveau optimal (membre de droite de (9)). Avec ces instruments, le choix décentralisé de $e$ et $z$ sera optimal. En effet, en seconde période l'individu choisira $e$ et $z$ de sorte que:

$$
z=\frac{w}{h\left(\ell^{*}, e\right)} \quad \text { et } \quad h_{2}\left(\ell^{*}, e\right) \frac{z^{2}}{2}=1 .
$$

\section{II-2 Optimum de Second Rang}

Nous supposons maintenant que l'autorité publique ne peut pas taxer ou subventionner l'épargne, soit parce qu'elle n'est pas directement observable, soit en raison d'éléments non modélisés, comme la mobilité internationale du capital. Ceci nous amène dans le cadre d'un optimum de second-rang. En d'autres termes, $\tau$ et $b$ poursuivent deux objectifs: réduire l'offre de travail à son niveau optimal et encourager l'épargne.

Nous devons d'abord étudier le choix de première période. L'individu myope doit trouver les valeurs effectives de $s$ et $\ell$ et anticipées (individuellement optimales ex ante) de $z$ et de $e$ qui maximisent:

$$
u\left(w(1-\tau) \ell-s-\frac{\ell^{2}}{2}\right)+u\left(w z+s+b-e-\frac{h(0, e) z^{2}}{2}\right) .
$$

On peut substituer dans cette expression $\ell=w(1-\tau)$ et écrire:

$$
u\left(\frac{w^{2}(1-\tau)^{2}}{2}-s\right)+u\left(w z+s+b-e-\frac{h(0, e) z^{2}}{2}\right) .
$$


La CPO pour le choix de $s$ (en l'absence de contrainte de liquidité) est simplement:

$$
u^{\prime}(\bar{c})=u^{\prime}\left(\bar{d}^{p}\right)
$$

On obtient ainsi une fonction d'épargne $s=s(\tau, b)$.

Notons que grâce à notre hypothèse de myopie extrême $(\alpha=0)$, les instruments $\tau$ et $b$ n'ont aucun effet sur le choix anticipé de $e$ et de $z$. Ces choix ex ante de $e$ et de $z$ sont différents des choix ex post, mais ils ne sont pas affectés par le choix de $\ell$. Par exemple, si l'on pose que $h(0, e)=1$, on en déduit que $z^{p}=w$ et $e^{p}=0$ et on obtient une valeur explicite de $s$ :

$$
s=\frac{w^{2}}{4}\left(\tau^{2}-2 \tau\right)-\frac{b}{2}
$$

avec

$$
\frac{\partial s}{\partial \tau}=\frac{w^{2}}{2}(\tau-1)<0, \frac{\partial s}{\partial b}=-\frac{1}{2}<0
$$

et $^{8}$

$$
\frac{\partial \tilde{s}}{\partial \tau}=\frac{\partial s}{\partial \tau}+\frac{\partial s}{\partial b} \frac{d b}{d \tau}=\frac{w^{2}}{2}(3 \tau-2),
$$

où la dernière égalité provient de la contrainte budgétaire: $b=\tau w \ell=\tau(1-\tau) w^{2}$. Remarquons que $\partial \tilde{s} / \partial \tau<0$ si $\tau<1 / 2$, c'est-à-dire si on se situe sur la partie croissante de la courbe de Laffer.

Le problème du planificateur consiste à choisir les valeurs de $\tau$ et de $b$ qui maximisent l'expression suivante:

$$
\begin{aligned}
\mathcal{L}= & u\left(\frac{w^{2}(1-\tau)^{2}}{2}-s\right)+u\left(s-e+\frac{w^{2}}{2 h(w(1-\tau), e)}+b\right) \\
& -\mu\left[b-\tau(1-\tau) w^{2}\right] .
\end{aligned}
$$

Dans cette expression, nous avons utilisé la valeur de $z$ que choisit l'individu dans sa deuxième période de vie, et qui est donnée par (5). Nous utilisons aussi la valeur de $e$ choisie en seconde période (équation (6)). Enfin, $\mu$ est le multiplicateur de Lagrange associé à la contrainte budgétaire de l'Etat.

8. Nous dénotons par un tilde la version compensée d'une variable. 
Les CPO s'écrivent:

$$
\begin{aligned}
\frac{\partial \mathcal{L}}{\partial \tau}= & -u^{\prime}(\bar{c})\left[w^{2}(1-\tau)+\frac{\partial s}{\partial \tau}\right]+u^{\prime}(\bar{d})\left[\frac{\partial s}{\partial \tau}+\frac{w^{3}}{2 h^{2}} h_{1}\right] \\
& +\mu(1-2 \tau) w^{2}=0, \\
\frac{\partial \mathcal{L}}{\partial b}= & u^{\prime}(\bar{c})\left[-\frac{\partial s}{\partial b}\right]+u^{\prime}(\bar{d})\left[\frac{\partial s}{\partial b}+1\right]-\mu=0 .
\end{aligned}
$$

Ces deux expressions peuvent être combinées en termes compensés:

$$
\begin{aligned}
\frac{\partial \widetilde{\mathcal{L}}}{\partial \tau} & =\frac{\partial \mathcal{L}}{\partial \tau}+\frac{\partial \mathcal{L}}{\partial b}(1-2 \tau) w^{2} \\
& =\Delta\left[\frac{\partial \tilde{s}}{\partial \tau}+w^{2}(1-\tau)\right]+u^{\prime}(\bar{d}) \frac{w^{3}}{2 h^{2}} h_{1}-u^{\prime}(\bar{d}) \tau w^{2}=0
\end{aligned}
$$

où $\Delta \equiv u^{\prime}(\bar{d})-u^{\prime}(\bar{c})$ reflète l'importance d'égaliser les niveaux de consommation dans le temps. Ainsi qu'il apparaît, cette équation comprend quatre termes qu'il nous faut signer.

Le myope égalise $\bar{c}$ et

$$
\begin{aligned}
\bar{d}^{p} & =w z^{p}-\frac{h\left(0, e^{p}\right)\left(\left(z^{p}\right)^{2}\right)}{2}-e^{p}+b+s \\
& =\frac{w z^{p}}{2}-e^{p}+b+s
\end{aligned}
$$

où la deuxième égalité est obtenue en utilisant l'équation (3). Dans le même temps, on a que

$$
\begin{aligned}
\bar{d} & =w z-\frac{h(w(1-\tau), e)\left(z^{2}\right)}{2}-e+b+s \\
& =\frac{w z}{2}-e+b+s
\end{aligned}
$$

où la deuxième égalité s'obtient grâce à (5). Comme on a $z<z^{p}$ et $e>e^{p}$, on a bien que $\bar{d}<\bar{d}^{p}=\bar{c}$ et donc que $\Delta>0$.

A partir de l'équation (10), on voit les quatre effets de la retraite et de la cotisation sur le bien-être de notre individu représentatif. Le premier effet représente l'incidence de la taxe sur l'épargne. Le signe de cet effet dépend du signe de $\partial \tilde{s} / \partial \tau$. Ainsi, si celui-ci est négatif, la taxe décourage l'épargne alors qu'on voudrait l'accroître, ce qui pousse à une taxe négative. Le second effet a un signe positif, car la retraite implique un transfert de la première à la seconde période qui est le bienvenu. Le troisième effet 
pousse également à une taxe positive en raison de son impact positif sur la désutilité du travail en seconde période. Enfin, le quatrième effet est négatif et souligne que la taxe implique une distorsion allocative qui est proportionnelle à l'élasticité de l'offre de travail (avec une fonction quadratique, on obtient $\partial \ell / \partial \tau=-w$.)

Le taux de taxation optimal $\tau$ est quant à lui donné par

$$
\tau=\frac{\Delta\left[\frac{\partial \tilde{s}}{\partial \tau} \frac{1}{w^{2}}+1\right]+u^{\prime}(\bar{d}) \frac{w}{2 h^{2}} h_{1}}{2 u^{\prime}(\bar{d})-u^{\prime}(\bar{c})} .
$$

Notons que si $\partial \tilde{s} / \partial \tau>0$, trois des effets mentionnés ci-dessus sont positifs et l'emportent sur le seul effet négatif car le taux optimal donné par (11) est positif. En revanche, si $\partial \tilde{s} / \partial \tau<0$, le signe de (11) est ambigu. Avec l'exemple ci-dessus, on obtient

$$
\frac{\partial \tilde{s}}{\partial \tau}=\frac{w^{2}}{2}(3 \tau-2)
$$

et avec $\tau=0$, nous avons

$$
\left.\frac{\partial \tilde{\mathcal{L}}}{\partial \tau}\right|_{\tau=0}=\frac{w^{3} u^{\prime}(d) h_{1}}{2 h^{2}}>0 .
$$

Plus généralement,

$$
\tau=\frac{u^{\prime}(\bar{d}) w \frac{h_{1}}{h^{2}}}{2 u^{\prime}(\bar{d})-\Delta}>0 .
$$

Naturellement, avec $h_{1}=0$, l'individu anticipe correctement ses choix de deuxième période, de sorte que $\Delta=0$ et $\tau=0$.

\section{Individus Différents}

Nous faisons maintenant l'hypothèse d'individus différents en productivité $w_{i}$. Tous sont myopes avec $\alpha=0$. Nous gardons les mêmes instruments, une retraite $b$ uniforme et une taxe linéaire $\tau$ sur le travail de première période. Le problème du planificateur social utilitariste et paternaliste peut s'exprimer par le Lagrangien suivant:

$$
\begin{aligned}
\mathcal{L}_{2}= & \sum n_{i}\left[u\left(\frac{w_{i}^{2}(1-\tau)^{2}}{2}-s_{i}\right)+u\left(\frac{w_{i}^{2}}{2 h\left(\ell_{i}, e_{i}\right)}-e_{i}+s_{i}+b\right)\right. \\
& \left.-\mu\left(b-\tau(1-\tau) \sum n_{i} w_{i}^{2}\right)\right]
\end{aligned}
$$


où $e_{i}$ est la valeur choisie par l'individu $i$ en seconde période (équation (6)). Rappelons que les valeurs anticipées de $e_{i}$ et $z_{i}$ sont indépendantes de $\tau$ et de $b$ et que $\ell_{i}=$ $w_{i}(1-\tau)$. Les CPO du planificateur social s'écrivent:

$$
\begin{aligned}
\frac{\partial \mathcal{L}_{2}}{\partial \tau}= & -E u^{\prime}(\bar{c})\left[w^{2}(1-\tau)+\frac{\partial s}{\partial \tau}\right]+E u^{\prime}(\bar{d})\left[\frac{w^{3}}{2 h^{2}} h_{1}(\ell, e)+\frac{\partial s}{\partial \tau}\right] \\
& +\mu(1-2 \tau) E w^{2}=0, \\
\frac{\partial \mathcal{L}_{2}}{\partial b}= & E u^{\prime}(\bar{c})\left[-\frac{\partial s}{\partial b}\right]+E u^{\prime}(\bar{d})\left[\frac{\partial s}{\partial b}+1\right]-\mu=0,
\end{aligned}
$$

où le symbole $E x$ représente l'espérance mathématique de la variable $x$ dans la population des individus $i$.

Comme plus haut, on combinera ces deux expressions pour obtenir des termes compensés.

$$
\begin{aligned}
\frac{\partial \tilde{\mathcal{L}}_{2}}{\partial \tau}= & \frac{\partial \mathcal{L}_{2}}{\partial \tau}+\frac{\partial \mathcal{L}_{2}}{\partial b}(1-2 \tau) E w^{2} \\
= & E \Delta \frac{\partial \tilde{s}}{\partial \tau}+E w^{2}\left[E \Delta(1-\tau)-\tau E u^{\prime}(\bar{d})\right]+E u^{\prime}(\bar{d}) \frac{w^{3}}{2 h^{2}} h_{1} \\
& -(1-\tau) \operatorname{cov}\left(u^{\prime}(\bar{c}), w^{2}\right)=0,
\end{aligned}
$$

où pour rappel $\Delta=u^{\prime}(\bar{d})-u^{\prime}(\bar{c})$.

Notons que dans le cas où tous les individus ont la même productivité, $\operatorname{cov}\left(u^{\prime}(\bar{c}), w^{2}\right)=$ 0 et l'on retrouve la formule (10). Par ailleurs, si $h_{1}=0$, il n'y a plus de problème de myopie $(\Delta=0)$. Dans ce cas, (12) se réduit à la formule de la taxation linéaire dans le cas où la désutilité du travail est quadratique:

$$
\frac{\tau}{1-\tau}=\frac{-\operatorname{cov}\left(u^{\prime}(\bar{c}), w^{2}\right)}{E u^{\prime}(\bar{d}) E w^{2}} .
$$

En général, on écrira:

$$
\begin{aligned}
\frac{\tau}{1-\tau}= & \frac{E w^{2} E \Delta-\operatorname{cov}\left(u^{\prime}(\bar{c}), w^{2}\right)}{E u^{\prime}(\bar{d}) E w^{2}}+\frac{E u^{\prime}(\bar{d}) w^{3} h_{1} / h^{2}}{2(1-\tau) E u^{\prime}(\bar{d}) E w^{2}} \\
& +\frac{E \Delta \partial \tilde{s} / \partial \tau}{(1-\tau) E u^{\prime}(\bar{d}) E w^{2}} .
\end{aligned}
$$

Cette expression se compose de trois termes. Le premier reprend la formule classique de la taxation linéaire des revenus, avec son dénominateur reflétant l'efficacité (il s'agit de la dérivée de l'offre de travail au revenu disponible) et le numérateur l'équité (cette 
covariance est négative). Le second terme est "pigouvien" et d'autant plus important que la durée du travail en première période affecte la capacité de travailler en seconde période. Le dernier terme reflète le besoin d'égaliser par l'épargne les utilités marginales des deux périodes. Les deux premiers termes sont positifs et le troisième négatif.

Par rapport à la section précédente, la formule (10) de la taxe optimale quand les individus sont identiques peut être exprimée comme

$$
\frac{\tau}{1-\tau}=\frac{w^{2} \Delta}{u^{\prime}(\bar{d}) w^{2}}+\frac{u^{\prime}(\bar{d}) w^{3} h_{1} / h^{2}}{2(1-\tau) u^{\prime}(\bar{d}) w^{2}}+\frac{\Delta \partial \tilde{s} / \partial \tau}{(1-\tau) u^{\prime}(\bar{d}) w^{2}} .
$$

L'introduction d'une hétérogénéité entre individus se traduit par un nouvel effet (lié à la covariance) qui tend à augmenter la taxe (le motif de redistribution). On s'attend donc à ce que la taxe optimale soit plus élevée dans ce dernier cas.

\section{Conclusion}

Dans cet article, nous avons montré que la taxation des revenus du travail pouvait être motivée, non seulement par des impératifs redistributifs, mais aussi par des considérations paternalistes: inciter les jeunes travailleurs à ménager leurs efforts afin d'être en bonne santé lorsqu'ils prennent de l'âge. Bien sûr, si les agents étaient parfaitement rationnels, ils prendraient les bonnes décisions et le seul but de la fiscalité serait redistributif. Nous nous sommes intéressés aux conséquences d'une certaine myopie quant aux effets de long terme. Nous avons utilisé le modèle le plus simple qui permette d'illustrer ce phénomène.

Il serait intéressant de généraliser ce modèle et d'introduire notamment une certaine hétérogénéité dans la myopie. En effet, tout le monde n'est pas myope et il est même possible que certains travailleurs, plutôt que d'ignorer l'effet qu'un travail trop long ou trop pénible peut avoir à terme sur leur capacité de poursuivre une vie active longue et épanouie, en viennent à le surestimer. Ces travailleurs pourraient choisir de travailler moins qu'il ne serait optimal et il faudrait les encourager à travailler davantage en première période et à épargner moins. Dans un optimum de premier rang, avec observabilité parfaite des types, à savoir des productivités et des degrés de myopie, il n'y a pas de problème de décentralisation. En cas d'asymétrie d'information, la politique 
optimale doit être faite de compromis et s'avérerait plus complexe que celle qui vient d'être présentée.

Un autre élément d'hétérogénéité qu'il faudrait prendre en compte est l'espérance de vie dont il a été question dans l'introduction. Dans cette introduction, nous avons rappelé que c'était surtout les personnes non qualifiées qui étaient concernées par la pénibilité du travail. Or, on sait que ces personnes ont une espérance de vie plus faible, en moyenne, que les travailleurs qualifiés (voir par exemple CAmBois et al. [2008]). On peut se demander si cette hétérogénéité est exogène ou si elle est (et dans quelle mesure) le résultat des choix faits sur le marché du travail par les individus.

A l'évidence, le modèle développé ici est très simple; il se concentre sur la question de choix de carrière limités au court terme et pour lesquels il y a un rôle pour une intervention publique. Nous nous rendons compte que de nombreux aspects de la réalité ne sont pas pris en compte pour des questions d'exposition. Par exemple, que faire de ceux qui choisissent de trop peu travailler, comme la cigale de la fable, ou de ceux dont la myopie concerne des biens "vertueux"? Il y a aussi dans ces illustrations place pour une intervention publique de nature paternaliste.

\section{Remerciements}

Nous remercions l'Agence Nationale de la Recherche pour son soutien financier dans le cadre du projet ANR "Retraite"(ANR-05-BLAN-0020). Nous remercions aussi les deux rapporteurs pour leurs excellents commentaires.

\section{Informations de contact}

\section{Helmuth Cremer}

Adresse: Toulouse School of Economics, Aile Jean-Jacques Laffont, 21 Allée de Brienne, F-31000, Toulouse, France

Email: helmuth.cremer@tse-fr.eu

Tel: $(+33)(0) 561128606$

Fax: $(+33)(0) 561128637$ 
Philippe De Donder (corresponding author)

Adresse: Toulouse School of Economics, MS102, 21 Allée de Brienne, F-31000, Toulouse, France

Email: dedonder@cict.fr

Tel: $(+33)(0) 561128542$

Fax: (+33) (0) 561128637

\section{Darío Maldonado}

Adresse: Carrera 5 15-37, Piso 5, 110321, Bogotá, Colombia

Email: dario.maldonado@urosario.edu.co

Tel: $(+571) 2970200 \times 8130$

Fax: $(+571) 3445763$

\section{Pierre Pestieau}

Adresse: Boulevard du Rectorat, 7 (B 31), B4000 - Liège - Belgique

Tel : (+32) 3663109

Fax: $(+32) 3662981$

Email : p.pestieau@ulg.ac.be

\section{Références}

[1] Blanchet, Didier et Thierry Debrand (2007): "Souhaiter Prendre sa Retraite le Plus Tôt Possible: Santé, Satisfaction au Travail et Facteurs Monétaires", Economie et Statistique, 403(1), p. 39-62. [1]

[2] Boisard Pierre, Damine Cartron, Michel Gollac et Antoine Valeyre (2002): Time and Work: Duration of Work. European Foundation for the Improvement of Living and Working Conditions. Dublin. [3] 
[3] Bound John, Michael Schoenbaum, Todd R. Stinebrickner et Timothy A. Waidmann (1999): "The Dynamic Effects of Health on the Labor Force Transitions of Older Workers", Labour Economics, 6(2), p. 179-202. [1]

[4] Cambois Emmanuelle, Caroline Laborde et Jean-Marie Robine (2008): "La "Double Peine" des Ouvriers: Plus d'Années d'Incapacité au Sein d'une Vie Plus Courte", Populations et Sociétés, 441. [15]

[5] Campolieti, Michele (2002): "Disability and the Labor Force Participation of Older Men in Canada", Labour Economics , 9(3), p. 405-432. [1]

[6] Caroli, Ève et Jérôme Gautié, (2009): Bas salaires et Qualité de l'Emploi: l'Exception Française? Paris: Ed. ENS rue d'Ulm. [I]

[7] Cremer, Helmuth, Jean-Marie Lozachmeur et Pierre Pestieau (2008): "Social Security and Retirement Decision: a Positive and Normative Approach", Journal of Economic Survey, 22 (2), p. 213-233. [1]

[8] Cremer, Helmuth, Philippe De Donder, Dario Maldonado et Pierre Pestieau (2010): "Taxing Sin Goods and Subsidizing Health Care", Scandinavian Journal of Economics (forthcoming). [3]

[9] Currie, Janet et Madrian Brigitte C. (1999): "Health, Health Insurance and the Labor Market", in Handbook of Labor Economics, Ashenfelter O. et Card D. éds., Amsterdam, North Holland, Chapter 50, p. 3309-3416. [I]

[10] Dahlgren, Anna (2006): "Work Stress and Overtime work: Effects on Cortisol, Sleep, Sleepiness and Health", Doctoral thesis, Department of Psychology, Stockholm University. [2, 3]

[11] De Donder, Phhilippe (2007): "Retraite et Myopie", in Les retraites. Libres opinions d'experts européens, sous la direction de Florence Legros, Economica, p. 247-254. [3]

[12] Debrand, Thierry et Pascale Lengagne (2007): "Pénibilité du Travail et Santé des Seniors en Europe", Economie et Statistique, 403-404, p. 19-38. [2]

[13] Dembe, Allard, J. Erickson, R. Delbos et S. Banks (2005): "The Impact of Overtime and Long Work Hours on Occupational Injuries and Illnesses: New Evi- 
dence From the United States", Occupational and Environmental Medecine, 62 (9), p. 588-597. [2]

[14] Dwyer Debra S. ex Olivia S. Mitchell (1999): "Health Problems as Determinants of Retirement: Are Selfrated Measures Endogenous?", Journal of Health Economics, 18 (2), p. 173-193. [1]

[15] Fenge, Robert et Pierre Pestieau (2005): Social Security and Retirement, MIT Press. [1]

[16] Hurd, Michael Et Kathlee McGarry (1995): "Evaluation of the Subjective Probabilities of Survival in the HRS", Journal of Human Resources, Special Issue: The Health and Retirement Study, Data Quality and Early Results, 30 (5), p. S268S292. [5]

[17] Hurd, Michael, James P. Smith et Julia M. Zissimopoulos (2004): "The Effects of Subjective Survival on Retirement and Social Security Claiming", Journal of Applied Econometrics, 19 (6), p. 761-775. [5]

[18] van Der Hulst, M (2003): "Long Workhours and Health", Scandinavian Journal of Work, Environment and Health, 29 (3), p. 171-188. [2]

[19] Karasek Robert ex Tores Theorell (1991): Healthy Work: Stress, Productivity, and the Reconstruction of Working Life, Basic Books, New York. [2]

[20] Kreider, Brent (1999): "Latent Work Disability and Reporting Bias", Journal of Human Resources, 34 (4), p. 734-769. [1]

[21] O’Donoghue, Ted et Matthew Rabin (2003): "Studying Optimal Paternalism, Illustrated by a Model of Sin Taxes", American Economic Review (Papers and Proceedings) 93 (2), p. 186-191. [3]

[22] O’Donoghue, Ted et Matthew Rabin (2006): "Optimal Sin Taxes", Journal of Public Economics, 90 (10-11), p. 1825-1849. [3]

[23] O'Donnell, Owen, Federica Teppa et Eddy van Doorslaer (2008): "Can Subjective Survival Expectations Explain Retirement Behaviour?", DNB Working Papers 188, Netherlands Central Bank, Research Department. [5] 
[24] OECD (2008): OECD Employment Outlook, Chapter 4: Are all jobs good for your health? The impact of work status and working conditions on mental health, Paris. $[2]$

[25] Siegrist J. (1996): "Adverse Health Effects of High-effort/Low-Reward Conditions", Journal of Occupational Health Psychology, 1 (1), p. 27-41. [2]

[26] Strotz, Robert H. (1956): "Myopia and Inconsistency in Dynamic Utility Maximization”, Review of Economic Studies, 23 (3), p. 165-180. [3]

0. Mots clés: Myopie, Retraite, Etat paternaliste, Biens peccamineux Codes JEL: D91, H21, H55 